\title{
REVIEW
}

Open Access

\section{Efficacy and safety of sprifermin injection for knee osteoarthritis treatment: a meta- analysis}

Ni Zeng ${ }^{1}$, Xin-Yuan Chen ${ }^{1}$, Zhi-Peng Yan ${ }^{1}$, Jie-Ting Li ${ }^{1}$, Tao Liao ${ }^{1}$ and Guo-Xin Ni ${ }^{1,2^{*}}$

\begin{abstract}
Objective: To perform a meta-analysis comparing the structural progression and clinical symptom outcomes as well as adverse events experienced from intra-articular injections of sprifermin compared to a placebo treatment for patients with knee osteoarthritis (KOA).
\end{abstract}

Method: We systematically searched the literature for studies that compared long-term outcomes between sprifermin and placebo injections for KOA treatment. Meta-analysis was performed with RevMan5.3 using an inverse variance approach with fixed or random effects models. Odds ratios (ORs) and 95\% confidence intervals (Cls) were estimated.

Results: Eight studies were included. Overall, there was significantly less improvement of WOMAC total scores in patients receiving sprifermin, compared with the placebo (mean difference $(M D)=3.23,95 \% \mathrm{Cl} 0.76-5.69 ; P^{2}=0 \%$; $P=0.01)$. Further, sprifermin injection patients gained more, and lost less, cartilage thickness and volume in total femorotibial joint (cartilage thickness: standardized mean differences $(S M D)=0.55,95 \% \mathrm{Cl} 0.26-0.84 ; P^{2}=78 \% ; P=$ 0.0002 ; cartilage volume: $\left.\mathrm{SMD}=0.39,95 \% \mathrm{Cl} 0.20-0.58 ; P^{2}=49 \% ; P<0.0001\right)$. Changes in the cartilage surface morphology of the medial tibio-femoral joint ( $\mathrm{MD}=-0.30,95 \% \mathrm{Cl}-0.44$ to $\left.-0.16 ; P^{2}=0 \% ; P<0.0001\right)$ and patellofemoral joint ( $\mathrm{MD}=-0.22 ; 95 \% \mathrm{Cl}-0.37$ to $\left.-0.07 ; P^{2}=0 \% ; P=0.004\right)$ showed a significant difference between the sprifermin and placebo injections. Moreover, there were no significant differences between sprifermin and the placebo in the risk of treatment-emergent adverse events $\left(\mathrm{OR}=1.05 ; 95 \% \mathrm{Cl} 0.52-2.14 ; P^{2}=48 \% ; P=0.89\right.$ ).

Conclusion: The data from the included studies provide strong evidence to determine the effect of intra-articular sprifermin on joint structure in individuals with KOA and show no specific adverse effects. Nevertheless, intraarticular sprifermin did not likely have any positive effect on symptom alleviation.

\footnotetext{
* Correspondence: niguoxin@bsu.edu.cn

'Department of Rehabilitation Medicine, The First Affiliated Hospital of Fujian Medical University, Fuzhou, China

${ }^{2}$ School of Sport Medicine and Rehabilitation, Beijing Sport University,

Beijing, China
} 


\section{Key messages}

- Intra-articular sprifermin is safe for the management of knee osteoarthritis.

- Intra-articular sprifermin may result in an improvement in cartilage thickness, volume, and surface morphology in KOA patients.

- Intra-articular sprifermin does not likely have any positive effect on symptom alleviation.

\section{Introduction}

Osteoarthritis (OA) is the most common type of arthritis and a leading cause of mobility-related disability [1]. It affects about $10 \%$ of those around the world who are 60 years and over and causes substantial economic burdens and socioeconomic consequences [1,2]. The symptoms of knee osteoarthritis (KOA), the knee being one of the most commonly affected joints, include limited range of motion, joint swelling, and pain that can cause disability [3].

Current therapies for OA are largely palliative and mainly focus on alleviating symptoms [4]. There are no pharmacological treatments known to prevent or cure OA [5]. International guidelines recommend the use of topical non-steroidal anti-inflammatory drugs (NSAIDs) and/or paracetamol as the first-line treatment of choice [3]. These drugs may relieve pain, but their prolonged consumption can result in severe adverse effects [3, 6]. Similarly, intra-articular injection of agents such as corticosteroids and hyaluronic acid may alleviate symptoms in KOA patients [7], but their effects on long-term clinical outcomes seem to be controversial [3, 7]. Given the nature of this chronic disorder, lifelong treatment should be required to arrest or slow its progression. Consequently, there is an urgent need for disease-modifying therapies that can alleviate symptoms and be safe for clinical use over long periods of time [8].

Sprifermin, a recombinant human fibroblast growth factor 18 , is currently being investigated as a potential disease-modifying OA drug [9]. Evidence has been accumulating to indicate that it could stimulate chondrocyte proliferation and increase knee joint cartilage thickness $[10,11]$. Recently, several placebo-controlled clinical trials $[12,13]$ have been conducted. While beneficial effects on the knee joint structure and clinical symptoms in KOA patients were reported in some studies [13, 14], others saw inconsistent results $[12,14]$. As such, an update on the current evidence is required. A meta-analysis was performed in this study to provide a much-needed and comprehensive assessment on the efficacy and safety of sprifermin injection and to evaluate its potential for clinical application in KOA patients.

\section{Methods}

The study protocol was registered with the International Prospective Register of Systematic Reviews (PROSPERO) (number CRD42020184508).

\section{Literature search}

A search of studies published between the start of each database and September 2020 was conducted using bibliographic databases, including PubMed, Embase, the Cochrane Library, and Ovid. We used a series of keyword combinations (Medical Subject Headings [MeSH]) and text terms in the titles and abstracts that described $\mathrm{OA}$ and sprifermin injection (full search strategy available in Supplement 1).

\section{Eligibility criteria}

Papers that met the following criteria were included in the analysis: (1) study design-RCT studies; (2) participants-patients with symptomatic knee $\mathrm{OA}$ at a Kellgren-Lawrence grade $\geqq 2$; (3) grouping-in addition to a therapy group with sprifermin injections, a control group receiving placebo treatments was included; (4) outcomes-outcomes reflecting efficacy (including symptoms, physical function, knee structure) and safety; and (5) language-published in English language journals.

The following were the exclusion criteria: (1) experimental studies (e.g., animal studies), (2) studies where the follow-up time is less than 1 month, (3) studies where sprifermin was combined with other drugs, (4) studies whose full text is not available, and (5) studies with no data available.

\section{Study selection and data extraction}

Two researchers (NZ and XYC) independently reviewed all the retrieved abstracts and full texts. If any disagreement was raised, it was resolved through discussion and consultation with a third researcher (ZPY). The following data were separately extracted by the two reviewers from the included studies: publication year, study design, number of participants, comparison group, treatment protocol of sprifermin, and outcome measures (such as the Western Ontario and McMaster Universities Osteoarthritis Index [WOMAC]), pain scores, cartilage thickness and morphology, treatment-emergent adverse events (TEAEs), and acute inflammatory reactions (AIRs).

\section{Risk of bias assessment}

Two researchers (NZ and XYC) independently evaluated the risk of bias in the included studies, using the Cochrane handbook $[15,16]$. Seven domains were evaluated: generation of randomization sequences, allocation concealment, blinding of participants and implementers, 
blinding of outcome assessment, incomplete outcome data, selective reporting, and other potential biases. The assessment of each domain was classified as low, high, or unknown risk of bias. For any disagreements, a third consultant (ZPY) was available to resolve the matter and elicit consensus. Studies involving three or more high risks of bias in the seven domains were considered to possess poor methodological quality.

\section{Statistical analysis}

Extracted data were analyzed using Review Manager V5.3. Continuous outcomes such as WOMAC pain scores, cartilage thickness, and cartilage volume were calculated and expressed as a weighted mean difference (WMD) (MD in RevMan V5.3) or as a standardized MD (SMD), while dichotomous data were expressed as an odds ratio (OR). A random-effects meta-analysis was used to compute a summary estimate, and a $95 \%$ CI was calculated for pooled estimates for each outcome.
Statistical significance was considered to be $P<0.05$. To assess heterogeneity between studies, $Q$ and $I^{2}$ statistics were calculated ( $P$ value less than 0.10 of the $Q$ statistics indicates heterogeneity, and a value of less than $50 \%$ of the $I^{2}$ statistics indicates low homogeneity, with a value of $75 \%$ or more indicating high heterogeneity) [17].

\section{Results}

\section{Search results}

Database searches initially identified 53 studies. After removing duplicates, 36 studies were reviewed by title and abstract. Sixteen papers were screened in full text, with eight papers meeting the eligibility criteria. The PRISMA flow chart for study screening at each step was established, as shown in Fig. 1.

\section{Study characteristics and quality assessment}

Characteristics of the included studies are listed in Table 1.

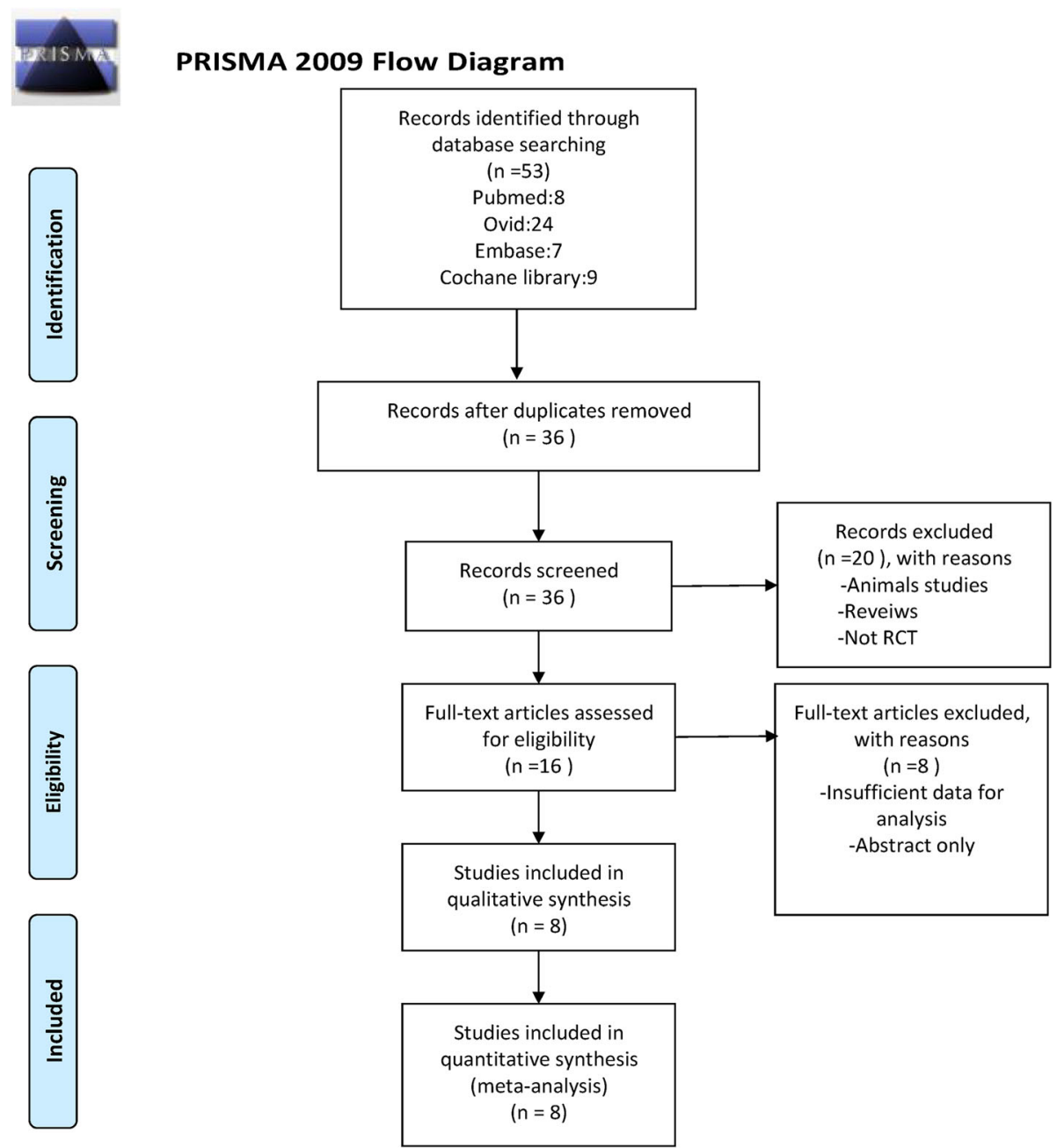

Fig. 1 Flow chart of the study's screening process 
Table 1 Characteristics of studies included in the meta-analysis

\begin{tabular}{|c|c|c|c|c|c|c|}
\hline Author (year) & Design & $\begin{array}{l}\text { Number of } \\
\text { patients }\end{array}$ & Sprifermin treatment strategy & Comparison & Follow-up & Outcomes \\
\hline Lohmander 2014 [18] & $\mathrm{RCT}$ & $n=192$ & $\begin{array}{l}\text { SAD/MAD } \\
\text { Doses: } 10,30,100 \mu \mathrm{g}\end{array}$ & Placebo & 12 months & $1,2,5,6,8,11$ \\
\hline Eckstein 2015 [19] & $\mathrm{RCT}$ & $n=168$ & $\begin{array}{l}\text { Injected } 3 \text { times over } 3 \text { weeks } \\
\text { Doses: } 10,30,100 \mu \mathrm{g}\end{array}$ & Placebo & 12 months & 6,7 \\
\hline Roemer 2020 [13] & $\mathrm{RCT}$ & $n=75$ & $\begin{array}{l}\text { SAD/MAD } \\
\text { Doses: } 10,30,100 \mu \mathrm{g}\end{array}$ & Placebo & 12 months & 9,10 \\
\hline Dahlberg 2016 [20] & $\mathrm{RCT}$ & $n=73$ & $\begin{array}{l}\text { SAD/MAD } \\
\text { Doses: SAD: 3, 10, 30, } 100 \mu \mathrm{g} \\
\text { MAD: } 3,10,30,100,300 \mu \mathrm{g}\end{array}$ & Placebo & 6 months & $1,2,3,4$ \\
\hline Roemer 2018 [21] & $\mathrm{RCT}$ & $n=549$ & $\begin{array}{l}100 \mu \mathrm{g} \mathrm{q} 6 \mathrm{mo}, 100 \mu \mathrm{g} \mathrm{q} 12 \mathrm{mo} \\
30 \mu \mathrm{g} \mathrm{q} 6 \mathrm{mo}, 30 \mu \mathrm{g} q 12 \mathrm{mo}\end{array}$ & Placebo & $6,12,18,24$ months & 9,10 \\
\hline Conaghan 2019 [22] & $\mathrm{RCT}$ & $n=549$ & $\begin{array}{l}100 \mu \mathrm{g} \mathrm{q} 6 \mathrm{mo}, 100 \mu \mathrm{g} \mathrm{q} 12 \mathrm{mo}, \\
30 \mu \mathrm{g} \mathrm{q} 6 \mathrm{mo}, 30 \mu \mathrm{g} 12 \mathrm{mo}\end{array}$ & Placebo & 2 years & 6 \\
\hline Hochberg 2019 [12] & $\mathrm{RCT}$ & $n=549$ & $\begin{array}{l}100 \mu \mathrm{g} \mathrm{q} 6 \mathrm{mo}, 100 \mu \mathrm{g} \mathrm{q} 12 \mathrm{mo}, \\
30 \mu \mathrm{g} \text { q6mo, } 30 \mu \mathrm{g} \mathrm{q} 12 \mathrm{mo}\end{array}$ & Placebo & 2 years $/ 3$ years & $1,2,3,4,5,6,8,12$ \\
\hline Eckstein 2020 [23] & $\mathrm{RCT}$ & $n=516$ & $\begin{array}{l}100 \mu \mathrm{g} \mathrm{q} 6 \mathrm{mo}, 100 \mu \mathrm{g} \mathrm{q} 12 \mathrm{mo}, \\
30 \mu \mathrm{g} \mathrm{q} 6 \mathrm{mo}, 30 \mu \mathrm{g} \mathrm{q} 12 \mathrm{mo}\end{array}$ & Placebo & 24 months & 6,7 \\
\hline
\end{tabular}

Outcomes: 1. treatment-emergent adverse events (TEAEs), 2. local TEAEs, 3. systemic TEAEs, 4. acute inflammatory reaction (AIRs), 5. Western Ontario and McMaster Universities Osteoarthritis Index (WOMAC), 6. cartilage thickness, 7. ordered values (OVs), 8. cartilage volume, 9. cartilage morphology, 10. bone marrow lesions (BMLs), 11. joint space width (JSW), and 12. minimum joint space width (mJSW)

$S A D$ single ascending dose, MAD multiple ascending dose, q6mo and q12mo sprifermin administered every 6 months and every 12 months, respectively

A total of eight studies were included in this metaanalysis [12, 13, 21], all published between 2014 and 2020, while there were only three original trials (NCT00911469 [Dahlberg 2016]; NCT01033994 [Lohmander 2014; Eckstein 2015; Roemer 2018]; NCT01919164 [Conaghan 2019; Hochberg 2019; Roemer 2020; Eckstein 2020]); the included studies analyzed different outcomes conducted on the three trials. All trials were comparison studies, including sprifermin and placebo, with a follow-up period of $\geqq 6$ months. The overall bias of the included studies is shown in
Fig. 2. Most studies were rated with a "moderate risk of bias."

\section{Efficacy of sprifermin injection for OA treatment Symptoms change measurements}

Change in WOMAC score from baseline Two studies (participants: sprifermin, $n=500$; Placebo, $n=127$ ) investigated the effect of sprifermin injection on symptom efficacy in KOA patients [12, 18], with Fig. 3 showing the outcomes of the meta-analysis for clinical symptoms

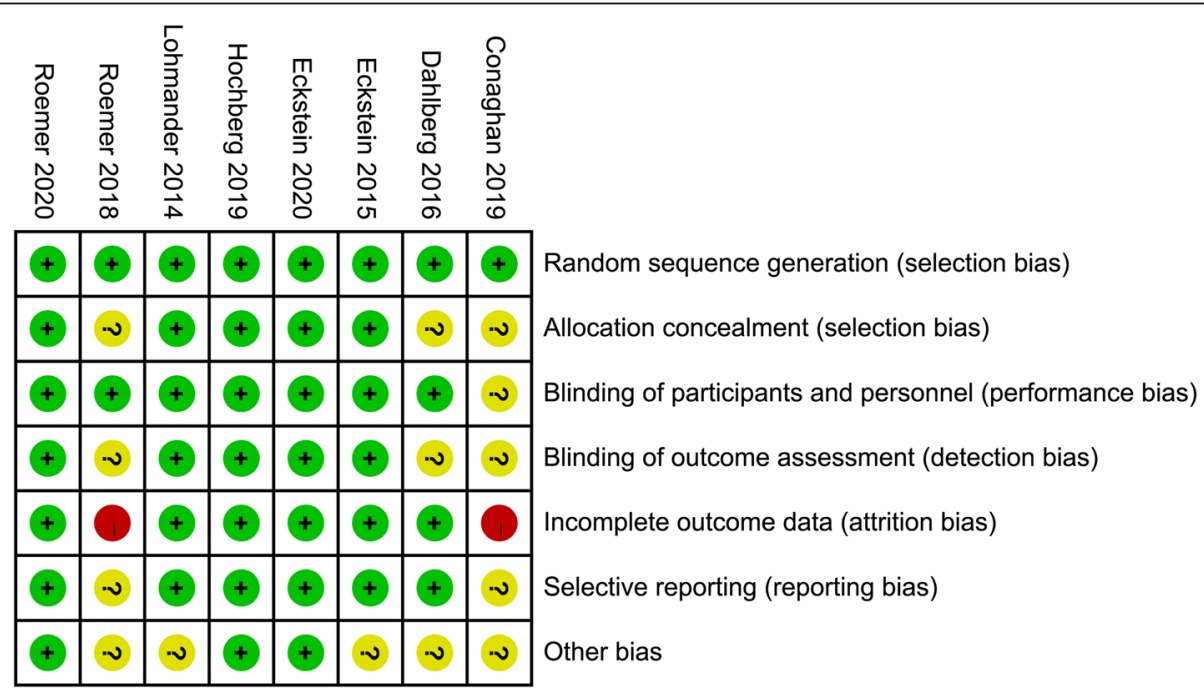

Fig. 2 Quality assessment of the included studies. The green background with " +" means low risk of bias; the red background with "-" means high risk of bias; the yellow background with "?" means unknown risk of bias 


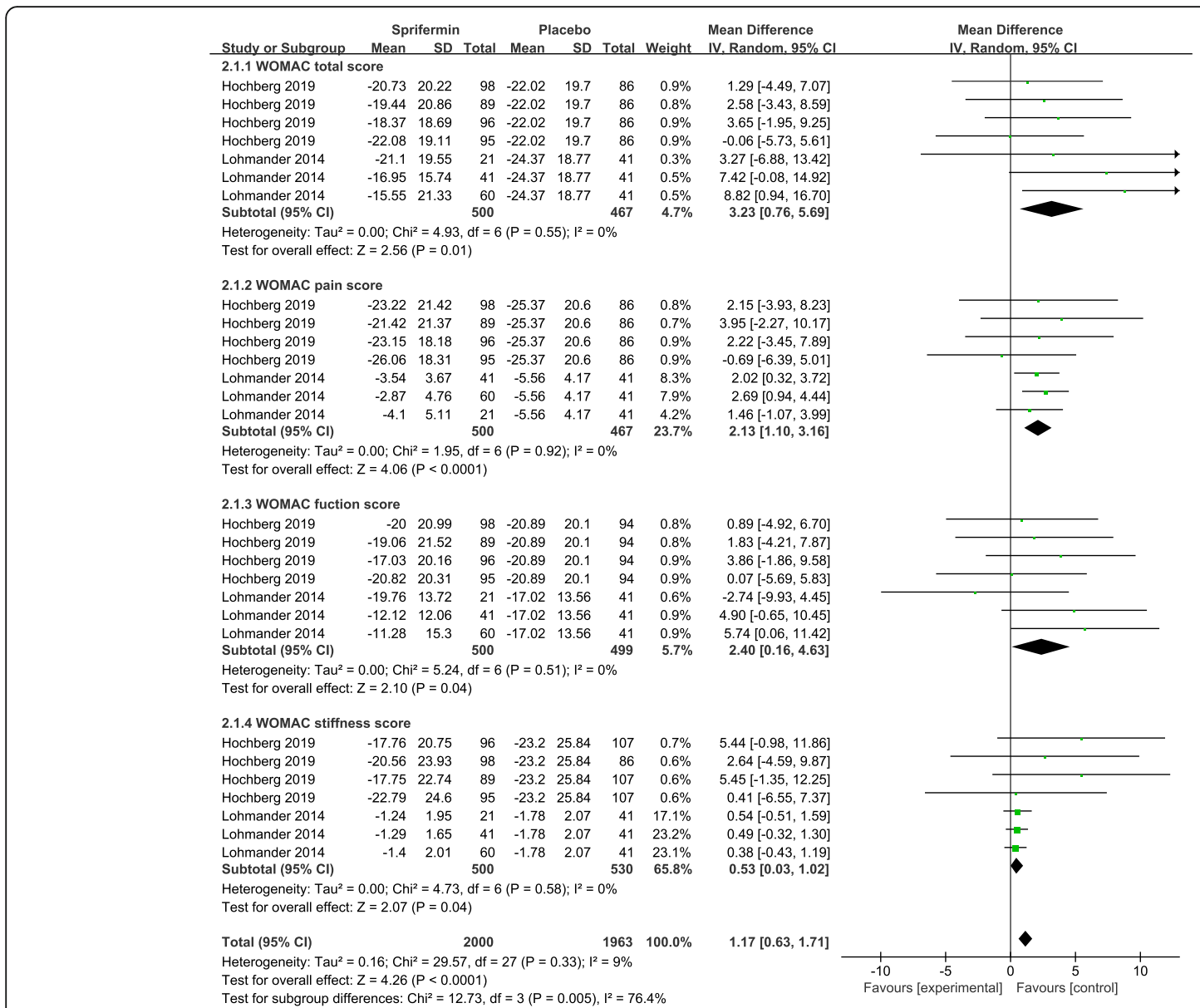

Fig. 3 Effectiveness for sprifermin versus placebo in symptom alleviation. Forest plots of the mean difference (MD) with 95\% confidence interval (CI) in WOMAC score (WOMAC total score, WOMAC pain score, WOMAC function score, WOMAC stiffness score) between patients undergoing sprifermin injection and placebo injection. (The three or four effect sizes for each trial in the figure represent the different dose of sprifermin treatment in the same trials)

and joint function. Symptom efficacy was evaluated as the change from baseline at 12 months [18] to 3 years [12] using WOMAC score. In comparison with the placebo group, sprifermin recipients exhibited less improvement in WOMAC scores, including for total $(\mathrm{MD}=3.23$, 95\% CI $\left.0.76-5.69 ; I^{2}=0 \% ; P=0.01\right)$, pain $(\mathrm{MD}=2.13$, $95 \%$ CI $\left.1.10-3.16 ; I^{2}=0 \% ; P<0.0001\right)$, function (MD = 2.40, 95\% CI $0.16-4.63 ; I^{2}=0 \% ; P=0.04$ ), and stiffness $\left(\mathrm{MD}=0.53,95 \%\right.$ CI $\left.0.03-1.02 ; I^{2}=0 \% ; P=0.04\right)$.

\section{Structural change measurements}

\section{Change in cartilage from baseline}

1. Cartilage thickness

Five RCTs were included for the meta-analysis of cartilage thickness management [12, 18-20, 22, 23]. Figure 3 shows a significant difference in the change from baseline in the total femorotibial joint (TFTJ) cartilage thickness of the experimental group (participants: $n=$
476) compared with the control group (participants: $n=$ 125) $\left(\mathrm{SMD}=0.55,95 \%\right.$ CI $0.26-0.84 ; I^{2}=78 \% ; P=$ 0.0002). Sprifermin-treated patients gained more, and subsequently lost less, cartilage thickness in the femorotibial subregions (lateral femorotibial compartments (LFTC), medial femorotibial compartment (MFTC), cMT, cLT, cMF, cLF) versus placebo-treated patients (Fig. 4). Also, five of 16 location-independent ordered values (OVs) of subregional change in cartilage thickness were analyzed, with the difference amounting to a statistical significance in all OVs assessed $(\mathrm{MD}=45.43,95 \%$ CI $36.69-54.18 ; I^{2}=0 \% ; \quad P<0.00001$ ) (eFigure 1 in Supplement 2).

\section{Cartilage volume}

Two studies (participants: sprifermin, $n=473$; Placebo, $n=124$ ) were included for meta-analysis of cartilage volume change $[12,18]$. As shown in eFigure 2 in Supplement 2, sprifermin injection led to higher rates of 


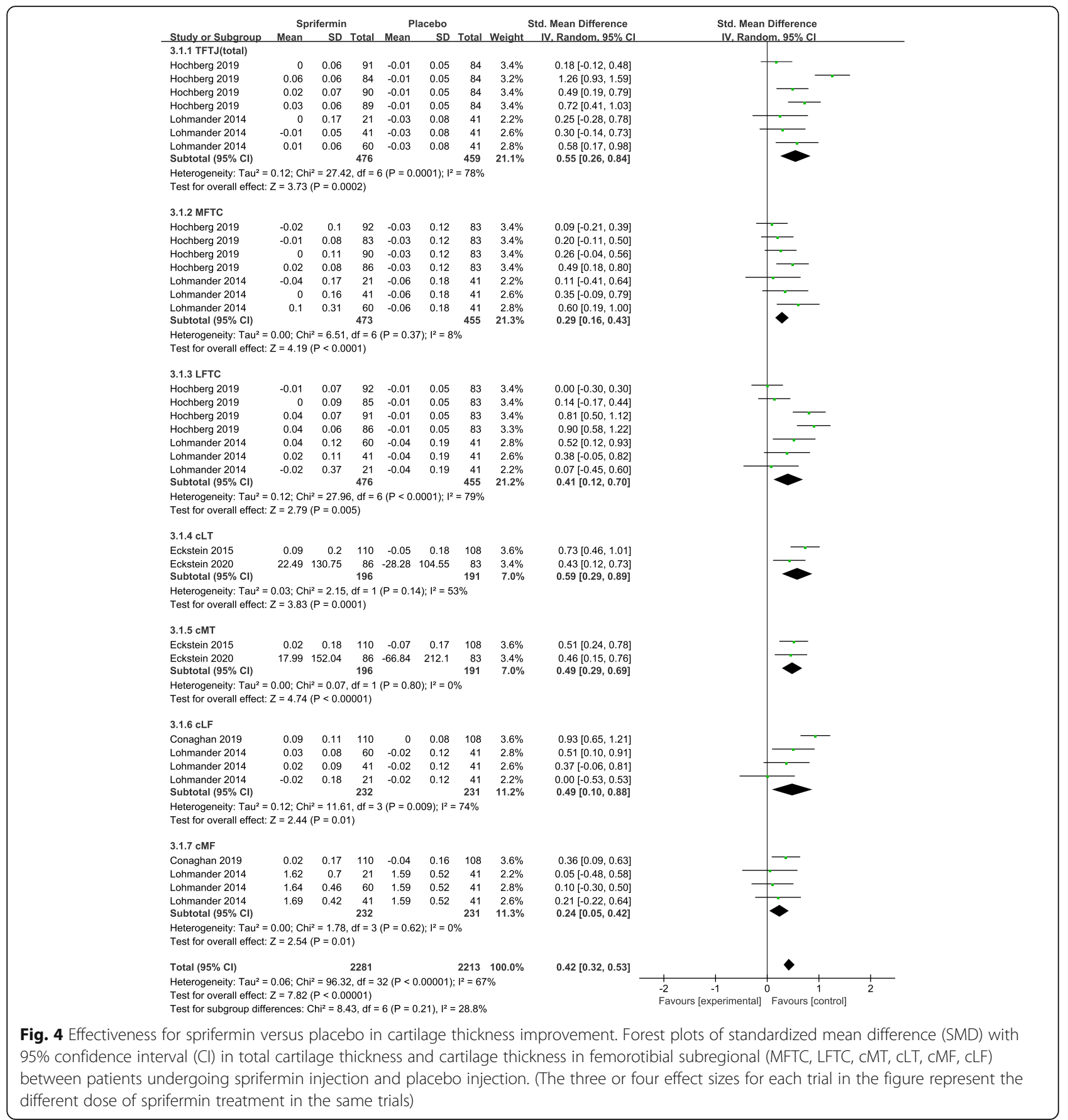

improvement of cartilage volume in the total knee region $\left(\mathrm{SMD}=0.39,95 \%\right.$ CI $0.20-0.58 ; I^{2}=49 \% ; \quad P<$ $0.0001)$ and LFTC $\left(\mathrm{SMD}=0.44,95 \%\right.$ CI $0.17-0.71 ; I^{2}=$ $75 \% ; P=0.001$ ), but not in MFTC (SMD:0.11, 95\% CI $-0.11-0.32 ; I^{2}=64 \% ; P=0.34$ ) when compared with placebo injection.

3. Cartilage morphology
Two studies (participants: sprifermin, $n=801$; placebo, $n=189$ ) evaluated the cartilage surface morphology using magnetic resonance images (MRI) [13, 21]. According to the results of the meta-analyses, cartilage surface morphology saw improvement in sprifermin-treated knees compared to placebo-treated knees, with effects being significant in the PFJ $(\mathrm{MD}=-0.22,95 \% \mathrm{CI}-0.33$ to $\left.-0.11 ; I^{2}=0 \% ; P=0.0001\right)$ and medial tibio-femoral joint 
(MFTJ) $\left(\mathrm{MD}=-0.30,95 \% \mathrm{CI}-0.44\right.$ to $-0.16 ; I^{2}=0 \%$; $P<0.0001)$, but not in the whole knee $(P=0.06)$ and lateral tibio-femoral joint (LFTJ) $(P=0.35)$ (eFigure 3 in Supplement 2).

Change in BMLs from baseline Two studies (participants: sprifermin, $n=800$; placebo, $n=190$ ) reported on bone marrow lesions (BMLs) using MRI [13, 21]. The application of sprifermin injection has no significant impact on the change of BMLs (included the whole knee and LTFJ, MTFJ, and PFJ) (eFigure 4 in Supplement 2).

Change in joint space width Two studies (participants: sprifermin, $n=517$; placebo, $n=128$ ) reported on joint space width (JSW) from radiographs $[12,18]$. Sprifermin was associated with statistically significant JSW narrowing in the lateral femorotibial compartment (MD 0.19, 95\% CI $0.09-0.30 ; I^{2}=44 \% ; P=0.0002$ ) (eFigure 5 in Supplement 2).

\section{Safety of sprifermin injections for osteoarthritis treatment}

Adverse events Three studies (participants: sprifermin, $n=639$; placebo, $n=173$ ) assessed the risk of overall and local TEAEs [12, 18, 20], and two (participants: sprifermin, $n=495$; placebo, $n=125$ ) assessed the risk of systemic TEAEs $[12,20]$. While the significantly decreased risk of systemic TEAEs was revealed with sprifermin injection $\left(\mathrm{OR}=0.56,95 \%\right.$ CI $0.37-0.87 ; I^{2}=0 \%$; $P=0.009)$, no significant differences were found in the risk of overall TEAEs $\left(\mathrm{OR}=1.05,95 \% \mathrm{CI} 0.52-2.14 ; I^{2}=\right.$ $48 \% ; P=0.89)$ or in the risk of local TEAEs $(\mathrm{OR}=1.25$, 95\% CI $0.52-2.96 ; I^{2}=42 \% ; P=0.62$ ) or between sprifermin and placebo injections, respectively (Fig. 5).

Acute inflammatory reaction Two studies (participants: sprifermin, $n=495$; placebo, $n=125)$ assessed the risk of acute inflammatory reaction (AIR) [12, 20]. There was no statistical difference in the risk of acute inflammatory reaction (AIR) between the sprifermin and placebo injection groups $(\mathrm{OR}=1.71,95 \%$ CI $0.95-3.07$; $\left.I^{2}=0 \% ; P=0.07\right)$ (eFigure 6 in Supplement 2).

\section{Discussion}

To the best of our knowledge, this is the first metaanalysis that aims to provide a comprehensive evaluation of the efficacy and safety of intra-articular sprifermin injections in KOA patients. The data from the included studies provided sufficient evidence to determine no specific adverse effects on the joint structure in individuals with KOA receiving intra-articular sprifermin injections. Nevertheless, its effect on symptom alleviation seems to be inconclusive.

Sprifermin is a potential disease-modifying OA drug for KOA $[3,9]$. Previous in vitro studies suggested its enhancement in chondrocyte proliferation and overall extracellular matrix production, as well as an increased repair response of mechanically damaged articular cartilage $[10,24]$. The data in the included RCT studies demonstrated that sprifermin has a beneficial effect on

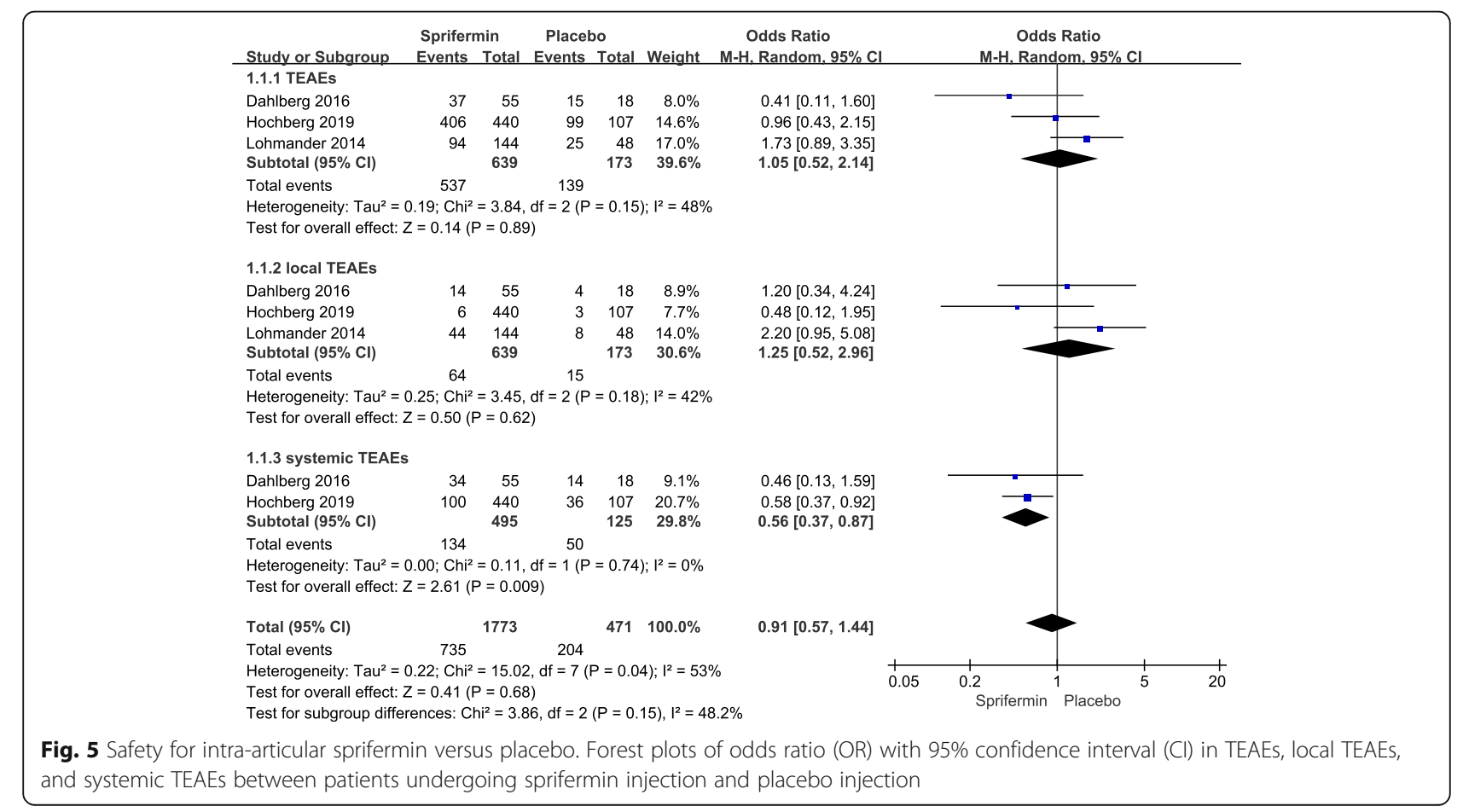


cartilage thickness, volume, and morphology in KOA patients, with the effect appearing to be location-specific. A preferential effect was revealed on either the lateral knee compartment or the medial compartment of the knee joint [18, 19, 23]. Since clinical trials may not account for clear differences in disease laterality, location-dependent effects on the cartilage would need further investigations [20]. However, it is worthwhile to note that dosage may be a crucial factor. Hochberg et al. reported that, in comparison with placebo, intraarticular sprifermin with $100 \mu \mathrm{g}$ of every 6 or 12 months induced a significant improvement in total femorotibial joint cartilage thickness, while intra-articular sprifermin with $30 \mu \mathrm{g}$ failed to induce a significant improvement [12]. On the other hand, in terms of BMLs, significant improvement was not found in the intra-articular sprifermin meta-analysis. The effects of sprifermin on subchondral BMLs, patterns that emerge from MRIs of the knees with $\mathrm{OA}$ and relate to structural and symptomatic progression of $\mathrm{OA}$, seem to largely depend on the duration, since the improvement of BMLs was observed at a 24-month follow-up [21], but not at 6 or 12 months [14, 21]. Further investigations are warranted to better understand the effects of intra-articular sprifermin on the improvement of BMLs.

Our data demonstrated that it is unlikely that intraarticular sprifermin leads to significant improvement in physical function and clinical symptoms in KOA patients. However, this finding should be taken with a grain of salt since the effects seem to change with time and in relation to patient baseline characteristics. When compared to the placebo, intra-articular sprifermin led to significantly less improvement at year 1 [18] with no statistical differences at years 2 and 3 [12], as well as a significantly greater improvement at year 3 in the "atrisk" subgroup (a patient subgroup with higher pain scores and lower joint space width at baseline) [25] among the WOMAC total scores. Further investigations are required to understand its long-term effect on clinical symptoms and functions in a targeted population. Additionally, even though DMOAD may not prevent pain in the latter stages of OA [18], its effect in preserving cartilage may prolong the time before KOA patients reach levels of debilitating pain $[12,18]$.

In addition to its efficacy, the safety of intra-articular sprifermin was also demonstrated in this study, suggesting that sprifermin holds potential for clinical application in KOA patients. Another important aspect to consider when interpreting the results is the study quality. Two included studies $[21,22]$ are conference abstracts, which makes it difficult to assess their quality. And Dahlberg et al. [20] only reported that participants were randomized but gave no explanation of the procedure and did not report the method used to conceal the allocation sequence in sufficient detail. All studies presented performance bias due to the impossibility of blinding personnel and participants. For example, interactive web/voice response systems were used to assign a blinded treatment kit number to each participant at each visit for the administration of sprifermin in the original trials (NCT01033994 and NCT01919164).

Nevertheless, this study has several limitations. First, only eight studies from three original trials were included in this study. As such, several key indicators were not analyzed in this study, like the visual analog scale. Another limitation relates to the heterogeneity of the outcome. Although statistical heterogeneity does not exist in most outcomes among the included studies $\left(I^{2}<50 \%\right)$, significant heterogeneity does exist in a couple of outcomes. For example, total cartilage thickness has an $I^{2}$ of $78 \%$. Subgroup analysis was not performed due to the limited articles included, which appears to compromise our findings. However, we suggested that the high level of heterogeneity among various studies is due to different treatment protocols (such as intra-articular dosage). When evaluating the change from baseline in total cartilage thickness of the sprifermin group compared with the control group, by including the outcomes of sprifermin with the same dosage (like $100 \mu \mathrm{g}$ ) instead of all treatment dosage of sprifermin, $I^{2}$ was changed from 78 to $0 \%$. Furthermore, most included studies were rated with "moderate risk of bias." The quality of these trials may reduce the confidence in the effect estimates observed in the present metaanalyses. More high-quality RCT trials are required to better understand the efficacy and safety of intraarticular sprifermin in the treatment of KOA patients.

\section{Conclusion}

The data from the included studies provides strong evidence to determine the effect of intra-articular sprifermin on the joint structure in individuals with KOA and shows no specific adverse effects. On the other hand, intra-articular sprifermin did not likely have any positive effect on symptom alleviation. While sprifermin can be regarded as a potential DMOAD for KOA patients, more evidence is still required for its efficacy and safety.

\footnotetext{
Abbreviations

AIRs: Acute inflammatory reactions; BMLs: Bone marrow lesions; Cls: Confidence intervals; JSW: Joint space width; KOA: Knee osteoarthritis; LFTC: Lateral femorotibial compartments; MD: Mean difference; MFTC: Medial femorotibial compartment; MeSH: Medical Subject Headings; MRI: Magnetic resonance images; mJSW: Minimum joint space width; NSAIDs: Non-steroidal anti-inflammatory drugs; ORs: Risk ratios; OVs: Ordered values; PFJ: Patellofemoral joint; RCTs: Randomized controlled trials; SMD: Standardized mean difference; TEAEs: Treatment-emergent adverse events; TFTJ: Total femorotibial joint; WOMAC: Western Ontario and McMaster Universities Osteoarthritis Index
} 


\section{Supplementary Information}

Supplementary information accompanies this paper at https://doi.org/10. 1186/s13075-021-02488-W.

Additional file 1. Search strategies for Pubmed, EMBASE, The Cochrane Library database and Ovid.

Additional file 2. Forest plots of intra-articular Sprifermin vs placebo on OVs, cartilage volume, cartilage morphology, BMLs, JSW and AIRs. Figure 1. Forest plots of mean difference (MD) with $95 \%$ confidence interval (Cl)in OVs between patients undergoing Sprifermin injection and placebo injection. Figure 2. Forest plots of standardised mean difference (SMD) with $95 \%$ confidence interval $(\mathrm{Cl})$ in total cartilage volume and cartilage volume in femorotibial subregional (MFTC, LFTC) between patients undergoing Sprifermin injection and placebo injection. (The three or four effect sizes for each trial in the figure represents different dose of Sprifermin treatment in the same trials). Figure 3. Forest plots of mean difference (MD) with 95\% confidence interval (Cl) in cartilage morphology of whole knee, MTFJ, LTFJ and PFJ between patients undergoing Sprifermin injection and placebo injection. (The three or four effect sizes for each trial in the figure represents different dose of Sprifermin treatment in the same trials). Figure 4. Forest plots of mean difference (MD) with 95\% confidence interval (Cl) in BMLs of whole knee, MTFJ, LTFJ and PFJ between patients undergoing Sprifermin injection and placebo injection. (The three or four effect sizes for each trial in the figure represents different dose of Sprifermin treatment in the same trials). Figure 5. Forest plots of mean difference (MD) with $95 \%$ confidence interval (CI) in JSW of medial and lateral femorotibial compartment between patients undergoing Sprifermin injection and placebo injection. (The three or four effect sizes for each trial in the figure represents different dose of Sprifermin treatment in the same trials).

Figure 6. Forest plots of odds ratio (OR) with 95\% confidence interval (CI) in AIRs, between patients undergoing Sprifermin injection and placebo injection.

\section{Acknowledgements}

N/A

\section{Authors' contributions}

All authors read and approved the final manuscript. And all authors discuss the concept of the manuscript. Conception or design of the work: NZ, GXN and XYC. Data collection: NZ and XYC. Data analysis and interpretation: NZ, GXN, XYC, ZPY, JTL, and TL. Drafting the article: NZ, XYP, ZPY, and TL. Critical revision of the article: NZ, GXN, and JTL.

\section{Funding}

This work was supported by the National Natural Science Foundation of China (81572219, 81871848).

\section{Availability of data and materials}

Not applicable.

\section{Declarations}

Ethics approval and consent to participate

Not applicable.

\section{Consent for publication}

All authors gave consent to publish.

\section{Competing interests}

The authors declare that they have no competing interests.

Received: 14 October 2020 Accepted: 22 March 2021

Published online: 09 April 2021

\section{References}

1. Appleton CT. Osteoarthritis year in review 2017: biology. Osteoarthr Cartil. 2018;26(3):296-303. https://doi.org/10.1016/j.joca.2017.10.008.

2. Hsieh JL, Shiau AL, Lee CH, Yang SJ, Lee BO, Jou IM, Wu CL, Chen SH, Shen PC. CD8+ T cell-induced expression of tissue inhibitor of metalloproteinses-
1 exacerbated osteoarthritis. Int J Mol Sci. 2013;14(10):19951-70. https://doi. org/10.3390/ijms141019951.

3. Hunter DJ, Bierma-Zeinstra S. Osteoarthritis. Lancet. 2019;393(10182):174559. https://doi.org/10.1016/S0140-6736(19)30417-9.

4. Van Spil WE, Kubassova O, Boesen M, Bay-Jensen AC, Mobasheri A. Osteoarthritis phenotypes and novel therapeutic targets. Biochem Pharmacol. 2019;165:41-8. https://doi.org/10.1016/j.bcp.2019.02.037.

5. Alcaraz MJ, Guillén MI, Ferrándiz ML. Emerging therapeutic agents in osteoarthritis. Biochem Pharmacol. 2019;165:4-16. https://doi.org/10.1016/j. bcp.2019.02.034

6. Tonge DP, Pearson MJ, Jones SW. The hallmarks of osteoarthritis and the potential to develop personalised disease-modifying pharmacological therapeutics. Osteoarthr Cartil. 2014;22(5):609-21. https://doi.org/10.1016/j. joca.2014.03.004

7. Huang Y, Liu X, Xu X, Liu J. Intra-articular injections of platelet-rich plasma, hyaluronic acid or corticosteroids for knee osteoarthritis: a prospective randomized controlled study. Der Orthopade. 2019;48(3):239-47. https://doi. org/10.1007/s00132-018-03659-5

8. Oo WM, Yu SP, Daniel MS, Hunter DJ. Disease-modifying drugs in osteoarthritis: current understanding and future therapeutics. Expert Opin Emerg Drugs. 2018;23(4):331-47. https://doi.org/10.1080/14728214.2 018.1547706

9. Marhardt K, Muurahainen N. Development of a disease-modifying OA drug (DMOAD) in knee osteoarthritis: the example of sprifermin. Drug Res (Stuttg). 2015;65(Suppl 1):S13.

10. Gigout A, Guehring H, Froemel D, Meurer A, Ladel C, Reker D, Bay-Jensen AC, Karsdal MA, Lindemann S. Sprifermin (rhFGF18) enables proliferation of chondrocytes producing a hyaline cartilage matrix. Osteoarthr Cartil. 2017; 25(11):1858-67. https://doi.org/10.1016/j.joca.2017.08.004.

11. Reker D, Kjelgaard-Petersen CF, Siebuhr AS, Michaelis M, Gigout A, Karsdal MA, Ladel C, Bay-Jensen AC. Sprifermin (rhFGF18) modulates extracellular matrix turnover in cartilage explants ex vivo. J Transl Med. 2017;15(1):250. https://doi.org/10.1186/s12967-017-1356-8.

12. Hochberg MC, Guermazi A, Guehring H, Aydemir A, Wax S, FleuranceauMorel P, Reinstrup Bihlet A, Byrjalsen I, Ragnar Andersen J, Eckstein F. Effect of intra-articular sprifermin vs placebo on femorotibial joint cartilage thickness in patients with osteoarthritis: the FORWARD Randomized Clinical Trial. JAMA. 2019:322(14):1360-70. https://doi.org/10.1001/jama.2019.14735.

13. Roemer F, Kraines J, Aydemir A, Wax S, Crema M, Hochberg M, et al. Evaluating the structural effects of intra-articular sprifermin on cartilage and non-cartilaginous tissue alterations, based on sqMRI assessment over 2 years. Osteoarthr Cartil. 2020;28(9):1229-34. https://doi.org/10.101 6/j.joca.2020.05.015.

14. Roemer FW, Aydemir A, Lohmander S, Crema MD, Marra MD, Muurahainen N, Felson DT, Eckstein F, Guermazi A. Structural effects of sprifermin in knee osteoarthritis: a post-hoc analysis on cartilage and non-cartilaginous tissue alterations in a randomized controlled trial. BMC Musculoskelet Disord. 2016;17(1):267. https://doi.org/10.1186/s12891-016-1128-2.

15. Wu Z, Ding $X$, Lei G, Zeng C, Wei J, Li J, Li H, Yang T, Cui Y, Xiong Y, Wang $Y$, Xie D. Efficacy and safety of the pulsed electromagnetic field in osteoarthritis: a meta-analysis. BMJ Open. 2018;8(12):e022879. https://doi. org/10.1136/bmjopen-2018-022879.

16. Cumpston M, Li T, Page MJ, Chandler J, Welch VA, Higgins JP, et al. Updated guidance for trusted systematic reviews: a new edition of the Cochrane handbook for systematic reviews of interventions. Cochrane Database Syst Rev. 2019;10:Ed000142.

17. Xia P, Wang X, Lin Q, Li X. Efficacy of mesenchymal stem cells injection for the management of knee osteoarthritis: a systematic review and meta-analysis. Int Orthop. 2015;39(12):2363-72. https://doi.org/10.1007/ s00264-015-2785-8

18. Lohmander LS, Hellot S, Dreher D, Krantz EFW, Kruger DS, Guermazi A, Eckstein F. Intraarticular sprifermin (recombinant human fibroblast growth factor 18) in knee osteoarthritis: a randomized, double-blind, placebocontrolled trial. Arthritis Rheumatol. 2014;66(7):1820-31. https://doi.org/10.1 002/art.38614

19. Eckstein F, Wirth W, Guermazi A, Maschek S, Aydemir A. Brief report: intraarticular sprifermin not only increases cartilage thickness, but also reduces cartilage loss: location-independent post hoc analysis using magnetic resonance imaging. Arthritis Rheumatol. 2015;67(11):2916-22.

20. Dahlberg LE, Aydemir A, Muurahainen N, Gühring $H$, Fredberg Edebo $H$, Krarup-Jensen N, Ladel CH, Jurvelin JS. A first-in-human, double-blind, 
randomised, placebo-controlled, dose ascending study of intra-articular rhFGF18 (sprifermin) in patients with advanced knee osteoarthritis. Clin Exp Rheumatol. 2016;34(3):445-50.

21. Roemer F, Kraines J, Aydemir A, Wax S, Crema M, Hochberg M, et al. Structural effects of intra-articular sprifermin in symptomatic radiographic knee osteoarthritis: a post-hoc analysis of cartilage and non-cartilaginous tissue alterations of the 2-year data from a5-year randomised, placebocontrolled, phase II study. Ann Rheum Dis. 2018;77;(Suppl. 2):795. https://doi. org/10.1136/annrheumdis-2018-eular.1960

22. Brett A, Bowes MA, Conaghan PG, Ladel C, Kraines J, Gühring H, et al. MRI data from the sprifermin phase II forward study: confirmation of manual cartilage segmentation findings by automated segmentation. Ann Rheum Dis. 2019;78:1421-2.

23. Eckstein F, Kraines JL, Aydemir A, Wirth W. Intra-articular sprifermin reduces cartilage loss in addition to increasing cartilage gain independent of location in the femorotibial joint: post-hoc analysis of a randomised, placebo-controlled phase II clinical trial. Ann Rheum Dis. 2020;79(4):525-8. https://doi.org/10.1136/annrheumdis-2019-216453.

24. Sennett ML, Meloni GR, Farran AJE, Guehring H, Mauck RL, Dodge GR Sprifermin treatment enhances cartilage integration in an in vitro repair model. J Orthop Res. 2018;36(10):2648-56. https://doi.org/10.1002/jor.24048.

25. Gühring H, Kraines J, Moreau F, Daelken B, Ladel C, Wirth W, et al. Cartilage thickness modification with Sprifermin in knee osteoarthritis patients translates into symptomatic improvement over placebo in patients at risk of further structural and symptomatic progression: post-hoc analysis of the phase ii forward trial. Ann Rheum Dis. 2019;78:70-1.

\section{Publisher's Note}

Springer Nature remains neutral with regard to jurisdictional claims in published maps and institutional affiliations. 